\title{
Biomarkers of Epidermal Innate Immunity in Premature and Full-Term Infants
}

\author{
VIVEK NARENDRAN, MARTY O. VISSCHER, IVAN ABRIL, STEPHEN W. HENDRIX, AND STEVEN B. HOATH \\ Department of Pediatrics [V.N., M.O.V., I.A., S.B.H.], University of Cincinnati College of Medicine, Cincinnati, Ohio 45229; The Procter \\ \& Gamble Company [S.W.H.], Cincinnati, Ohio 45267
}

\begin{abstract}
Epidermal innate immunity is a complex process involving a balance of pro- and anti-inflammatory cytokines, structural proteins, and specific antigen presenting cells occurring against a background of neuroendocrine modulators such as cortisol. In this study, a multiplex array system was used to simultaneously determine multiple molecular factors critical for development of epidermal innate immune function from the skin surface of premature and term infants, healthy adults, and vernix caseosa. Samples were analyzed for Keratin 1,10,11, Keratin 6, involucrin, albumin, fibronectin and cortisol, and cytokines IL-1, TNF $\alpha$, IL-6, IL-8, MCP1, IP10, IFN $\gamma$, and IL-1 receptor antagonist. Keratin 1,10,11 was decreased and involucrin was increased in infants versus adults. All infants had elevated IL $1 \alpha$ and reduced TNF $\alpha$ versus adults. IL-6, IL- 8 , and MCP1 were significantly increased in premature versus term infants and adults. Skin surface cortisol and albumin were significantly elevated in premature infants. The biomarker profile in premature infants was unique with differences in structural proteins, albumin, and cytokines IL-6, IL-1 $\beta$, IL-8, and MCP1. The higher infant IL1 $\alpha$ may be associated with skin barrier maturation. The significant elevations in skin surface cortisol for preterm infants may reflect a neuroendocrine response to the stress of premature birth. (Pediatr Res 67: 382-386, 2010)
\end{abstract}

$\mathrm{E}^{\mathrm{p}}$ pidermal innate immunity is a complex process involving a balance of pro- and anti-inflammatory cytokines, structural proteins, and specific antigen presenting cells. Orchestration of these factors occurs against a background of neuroendocrine modulators, e.g. cortisol. During the third trimester of pregnancy, an extraordinary process of epidermal differentiation culminates in formation of an intact environmental and innate immune interface, the stratum corneum (SC). The full-term infant relies on this protective interface during transition to a cold, dry, microbe-rich extrauterine environment at birth. In contrast, the premature infant is poorly equipped to handle such environmental stressors. The preterm epidermis is thinner, the immature SC has fewer cornified layers, and the "wounded" skin surface is more permeable. Poor SC integrity puts the premature infant at risk for high water loss, electrolyte imbalance, thermal instability, increased exposure to infectious agents, and environmental irritants due to increased permeability. The barrier develops

Received July 31, 2009; accepted November 19, 2009.

Correspondence: Marty O. Visscher, Ph.D., The Skin Sciences Institute, Cincinnati Children's Hospital Medical Center, 3333 Burnet Avenue, Cincinnati, OH 45229; e-mail: Marty.Visscher@cchmc.org

This project was a joint collaboration between Cincinnati Children's Hospital Medical Center and The Procter \& Gamble Company. Each entity provided funding for their investigators. rapidly, but 1 month later still has higher transepidermal water loss (TEWL) than term infants (1). Estimates of the time to complete barrier maturation vary from 2- to 9-wk postnatal age $(1,2)$.

In the classical stress response, the hypothalamus releases $\mathrm{CRH}$ (corticotrophin releasing hormone), which binds to corticotropin releasing hormone receptor 1 (CRH-R1) to stimulate release of adrenocorticotropic hormone (ACTH). ACTH stimulates the adrenal glands to produce cortisol, which acts to inhibit production of CRH. Remarkably, the skin itself contains all the elements of the hypothalamic-pituitary-adrenal (HPA) axis $(3,4)$. Understanding the function(s) of this peripheral system will require considerably more research but its putative purpose is to respond in part to local stressors, e.g. UV radiation, irritants, temperature, barrier disruption, and faced by the premature infant.

In addition to cortisol, the skin produces corticotropin releasing hormone (CRH) and receptors CRH-R1 and R2 (3). Melanocytes express CRH, CRH-R1, and proopiomelanocortin (POMC) and treatment with $\mathrm{CRH}$ activates $\mathrm{CRH}-\mathrm{R} 1$ to increase cAMP levels, expression of POMC genes, and ACTH production. ACTH and CRH lead to increases in cortisol and corticosterone (5). The epithelium of human hair follicles appears particularly important and manifests a fully functional peripheral equivalent of the HPA axis (6). Follicles in culture produce cortisol and levels are up-regulated on exposure to $\mathrm{CRH}$ and ACTH. CRH stimulates hair growth, keratinocyte proliferation, and production of hair pigment (melanin). Thus, hair follicles may serve as an extra-adrenal site of cortisol synthesis using complex regulatory feedback loops, similar to the classical HPA axis and, hypothetically, can operate locally in the skin as coordinator and executor of peripheral stress responses. Similarly, there is a CRH system in human sebocytes that has been implicated in acne (7). Corticosteroids can be produced from cholesterol in the skin (8). Collectively, reports suggest that $\mathrm{CRH}$ from cutaneous sources may exert both proinflammatory and anti-inflammatory effects $(9,10)$.

Innate immunity is also conferred by the SC via antiinfective lipid components, antimicrobial host defense proteins and a direct physical barrier (11). Sebum and sweat contribute protective factors, e.g. alpha tocopherol, to the skin surface. Epidermal keratinocytes secrete cytokines, such as

\footnotetext{
Abbreviations: CRH, corticotrophin releasing hormone; HPA, hypothalam-
} ic-pituitary-adrenal; SC, stratum corneum 
IL1, in response to SC damage and coordinate the recovery processes (12). In summary, the skin neuroendocrine system can potentially mount a progressive, intensity-dependent, highly coordinated stress response to a variety of noxious stimuli. We conducted an initial elucidation of the ontogeny of this skin system by evaluating premature infants $\leq 32$-wk GA, i.e. before the time of complete SC maturation, relative to full term infants and adults.

Central to this approach is the application of a new noninvasive sampling method (13), which simultaneously determines multiple factors critical for the development of epidermal innate immune function. The technique analyzes biomarkers from the outermost SC eliminating the need for invasive tissue biopsies. We evaluated a specific group of biomarkers in cohorts of infants and adults. We determined levels in vernix, a complex, topical, fetal-derived skin cream with innate immune and protective functions (14) that is generally sparse in very premature infants. We measured Keratin 1,10,11, Keratin 6, and involucrin to assess differences in SC maturation and quantified levels of albumin, fibronectin, cortisol, and cytokines IL1 $\alpha, \operatorname{IL} 1 \beta, \mathrm{TNF} \alpha$, IL6, MCP1, IL8, IL1RA, INF $\gamma$, and IP10.

\section{MATERIALS AND METHODS}

Subjects. Twenty full-term neonates (Christ Hospital, Cincinnati, OH) were enrolled within $2 \mathrm{~d}$ of birth. Nineteen premature infants $\leq 32$-wk GA (University Hospital, Cincinnati, OH) were enrolled within $7 \mathrm{~d}$ of birth. Twenty adults served as controls. The Institutional Review Board of Cincinnati Children's Hospital Medical Center approved the research and subjects provided written informed consent.

Skin surface sample collection. Duplicate samples were collected with $380 \mathrm{~mm}^{2}$ D-Squame tapes (CuDerm, Dallas, TX) applied to the forehead with consistent pressure, removed after $2 \mathrm{~min}$ and stored at $-80^{\circ} \mathrm{C}$. The skin was not pretreated before sampling at least $2 \mathrm{~h}$ after water exposure.

Vernix. Vernix was harvested at delivery from the skin surface of 11 (11) healthy term newborns and stored at $4^{\circ} \mathrm{C}$. Vernix was spread onto plastic slides and samples collected with D-Squame tapes.

Biomarker analysis. Biomarkers were extracted in PBS buffer, 0.2\% SDS, and $0.5 \%$ propylene glycol and sonicated for $60 \mathrm{~min}$ at $4^{\circ} \mathrm{C}$. Keratin $1,10,11$ (as a mixture), keratin 6, involucrin, fibronectin, cortisol, and albumin were quantified using Human Skin Panel Lincoplex Kit microsphere beads (Linco Research, Inc., St. Charles, MO) (13). IL1 $\alpha$, IL1 $\beta$, TNF $\alpha$, IL6, MCP1, IL8, IL1RA, INF $\gamma$, and IP10 were determined using human cytokine bead-based arrays (Linco Research) with a Bio-Plex multiplex suspension system (BioRad Laboratories, Hercules, CA). Total soluble protein was determined with a modified Lowry assay (porcine gelatin standard). Biomarkers were normalized to protein and reported as $\mathrm{ng}$ or $\mathrm{pg} / \mu \mathrm{g}$.

Statistical analysis. Data were analyzed with SigmaStat and SPSS (SPSS, Inc., Chicago, IL) with a significance level of $p<0.05$. Results are reported as mean \pm SEM. Univariate general linear models with least significant differences were used to compare the four groups. Covariates included GA, gender, race, structural proteins, and interactions. Relationships among biomarkers and with age were determined using Pearson or Spearman correlations.

\section{RESULTS}

Subjects. The full-term infants were of $38.6 \pm 1.2 \mathrm{wk}$ mean GA (Table 1). The premature infants were $\leq 32$-wk GA (mean $28.1 \pm 2.5$ ) with 10 from 24 to $27 \mathrm{wk}$ and 9 from 28 to $32 \mathrm{wk}$ (Table 1).

Structural proteins. Keratin 1,10,11 was higher in adults than in infants and vernix (GLM, $F=19.1, p<0.001$ ) (Fig. 1A). Keratin 6 was higher in preterms and adults than vernix (data not shown). The K6 to K1,10,11 ratio was higher in both infant groups versus adults $(F=7.1, p<0.001)$ (Fig. $1 B)$.
Table 1. Subject and sample demographics

\begin{tabular}{lcccc}
\hline & Premature & Full term & Adult & Vernix \\
\hline Number & 19 & 20 & 20 & 12 \\
GA, wk (mean SD) & & & N/A & N/A \\
Range & $(24-32)$ & $(36-40)$ & & \\
Gender & 5 & & & \\
$\quad$ Female & 14 & 11 & 8 & 5 \\
$\quad$ Male & 10 & 9 & 9 & 7 \\
Ethnicity & 8 & 9 & 3 & 1 \\
$\quad$ Caucasian & 1 & 2 & 8 & 2 \\
$\quad$ African American & & & & \\
$\quad$ Other & & &
\end{tabular}

N/A, not applicable.

Involucrin was higher in the preterms than all others and higher in full terms than adults $(F=44.5, p<0.001)$ (Fig. $1 C)$. Involucrin was inversely correlated with GA among the infants (correlation coefficient $=-0.77, p<0.001$ ).

Human albumin was higher in preterms than all others and higher in vernix than adults $(F=17.2, p<0.001)$ (Fig. $2 A)$. $\mathrm{SC}$ albumin was inversely related to GA, i.e. higher in the more premature infants $(r=-0.72, p<0.001)$. Fibronectin was lower in preterms and vernix versus adults $(F=4.5, p=$ 0.007 ) (Fig. 2B). Skin surface cortisol was higher in preterms than all others and vernix cortisol was lower than all others $(F=7.8, p<0.001)$. Cortisol was comparable for full terms and adults (Fig. 2C).

Cytokines. The proinflammatory cytokine IL $1 \alpha$ was higher in both infant groups versus adults and vernix $(F=11.5, p<$ 0.001 ) (Fig. $3 A$ ). With GA as a covariate, IL1 $\alpha$ was higher in premature than term infants. In contrast, $\mathrm{TNF} \alpha$ was significantly lower in both infant groups and vernix versus adults $(F=10.4, p<0.001)$ (Fig. $3 A)$. IL1 $\beta$ was higher in preterms than all others and higher in adults than vernix $(F=10.1, p<$ 0.001 ) (Fig. 3B). MCP1 was higher in preterms than full terms and vernix, whereas adult levels were higher than vernix $(F=$ 6.2, $p=0.001$ ) (Fig. 3B). INF $\gamma$ and IP10 were lower for vernix than for all others $(F=8.3, p<0.001 ; F=3.3, p=$ 0.03 , respectively). No differences were found for INF $\gamma$ or IP10 (data not shown).

The proinflammatory cytokine IL8 was higher in preterms than all others, which did not differ $(F=5.0, p=0.003)$ (Fig. $3 C$ ). IL6 was higher in preterms than in full terms and vernix, but not different from adult levels $(F=3.7, p=0.02)$ (Fig. 3C). IL8 and IL6 were moderately correlated with GA $(r=-0.44, p=$ 0.005 and $-0.33, p=0.04)$, with higher levels in younger infants. IL-1 receptor antagonist was not detectable in $21 \%$ of the preterm samples, $45 \%$ of full terms, $65 \%$ of adults, and $66 \%$ of vernix. IL1RA was detected in fewer preterms than adults $(z=$ $2.45, p=0.01)$ and vernix samples $(z=2.13, p=0.03)$.

\section{DISCUSSION}

The overall aim of this study was to evaluate the relevance and utility of measuring selected biomarkers of innate immune function from the skin surface of neonates and adults as a method for distinguishing ontogenetic groups. The results allow provisional discussion of the development of normal innate epidermal immune function and other protective mech- 

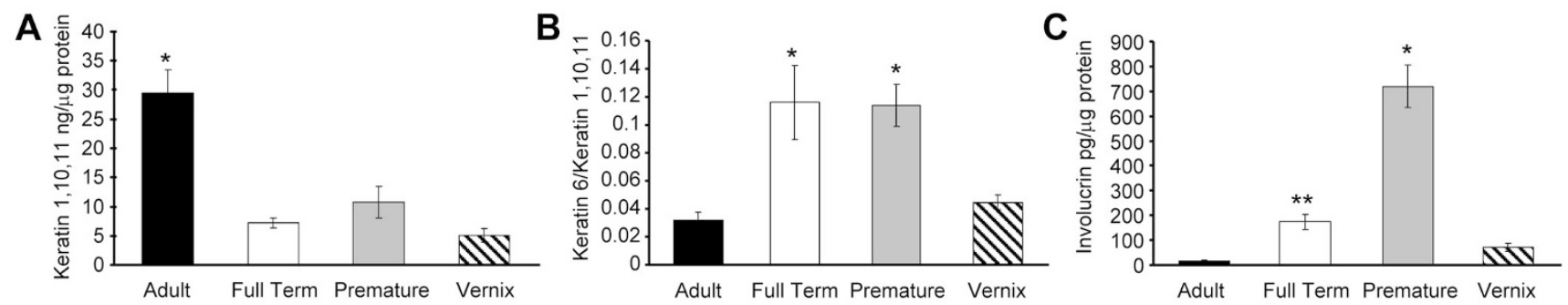

Figure 1. Structural proteins. $A$, Keratin $1,10,11$ was higher in adults than preterms, full terms and vernix ( $p<0.001)$. *Difference from all. $B$, Keratin 6 was higher in preterm and adults than vernix, but the K6 to K1,10,11 ratio was higher in both infant groups $v s$ adults $\left(^{*}\right)(p<0.001)$. $C$, Involucrin was higher in preterms than all (*) and higher in full terms than adults $(* *)(p<0.001)$.
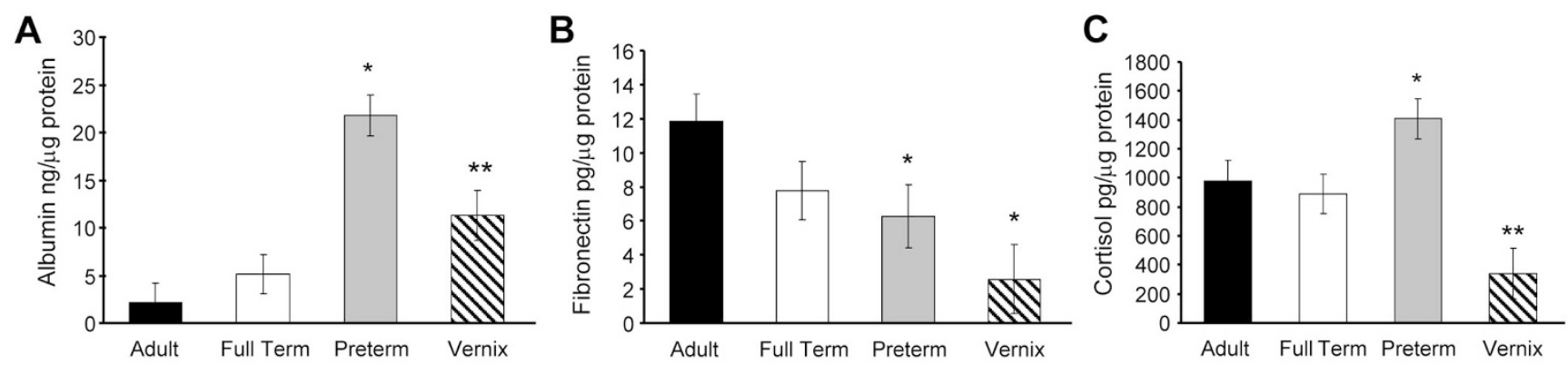

Figure 2. Albumin, fibronectin, and cortisol. A, Albumin was higher in preterms than all others $(*)$ and higher in vernix than adults $(* *)(p<0.001) . B$, Fibronectin was lower in preterm and vernix $v s$ adults $(p=0.007)$. $C$, Cortisol was higher in preterms than in all others $(*)$ and lower in vernix than all others $(* *)(p<0.001)$. Cortisol was comparable in full terms and adults.
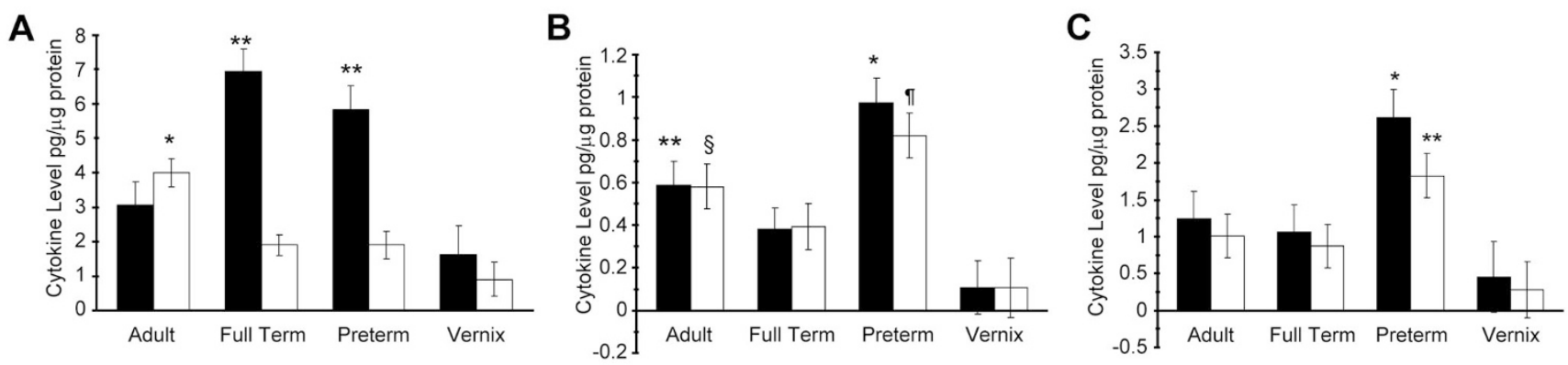

Figure 3. Cytokines. $A, \operatorname{IL} 1 \alpha(\square)$ was higher in both infant groups than in adults and vernix $(* *)(p<0.001)$. TNF $\alpha$ ( $\square)$ was lower in both infant groups and vernix vs adults $(*)(p<0.001) . B, \operatorname{IL} 1 \beta(\square)$ was higher in preterms than all others $(*)$ and higher in adults than vernix $(* *)(p<0.001)$. MCP1 $(\square)$ was higher in preterms than full terms and vernix (II) and adult levels were higher than vernix ( $\S)(p=0.001)$. $C$, IL8 ( $\square$ ) was higher in preterms $v s$ all others, which did not differ from each other $\left(^{*}\right)(p=0.003)$. IL6 $(\square)$ was higher in preterms than full terms and vernix, but not different from adults $(* *)(p=0.02)$.

anisms associated with environmental stress and postnatal adaptation. Significant differences were observed in the levels of biomarkers of epidermal innate immunity in premature infants compared with full-term newborns, adults, and the intrauterine (fetal) skin surface (vernix caseosa). Neonates $\leq 32$-wk GA had significantly higher levels of involucrin, albumin, proinflammatory cytokines IL1 $\beta$, IL6, MCP1, and IL8 than full-term infants and adults (Figs. $1 C, 2 A, 2 C, 3 B$, and $3 C$ ). Both infant groups had significantly higher IL $1 \alpha$ and Keratin 6/Keratin 1,10,11 than adults and significantly lower Keratin $1,10,11$ and TNF $\alpha$ than adults (Figs. $1 A, 1 B, 3 A$ ). Involucrin was higher in full terms than adults. Involucrin and albumin levels were inversely related to GA, most likely indicative of age effects on barrier maturation. Except for albumin, vernix had the lowest biomarker levels. Premature infants had higher levels of cortisol, putatively reflecting an increased stress response.

Keratin 1,10,11 was significantly lower in both infant groups than adults. Reduced keratin 1,10,11 has been associated with higher skin dryness (15) and chronic hyperproliferation (16). Full-term infants exhibit low SC hydration at birth followed by an increase during the first month (17). In preterms, barrier formation is rapid and exhibits abnormal desquamation indicative of SC hyperproliferation (18). Involucrin is an SC precursor that undergoes crosslinking to become part of the cornified envelope (19). SC with involucrin positive and fragile cornified envelopes has been associated with barrier impairment and inflammation, e.g. psoriasis vulgaris and atopic dermatitis (20). Early involucrin expression was linked with barrier disruption (21). 
Whereas Rabilloud et al. (22) reported albumin in the suprabasal epidermis and proposed diffusion through the basement membrane, Hasse et al. (23) found albumin in epidermal keratinocytes and suction blister roofs, confirming epidermal synthesis versus transport from serum. Albumin was significantly higher in lesional than uninvolved atopic skin and both were higher than nonatopic controls (24). Albumin was positively correlated with TEWL (lesional skin) and negatively correlated to skin hydration (uninvolved skin). This is consistent with our finding of increased albumin in infants $\leq 32 \mathrm{wks}$ and with low hydration shortly after birth (17). Albumin binds unsaturated fatty acids and calcium and may be involved in their transport (23). Its protective effects against $\mathrm{H}_{2} \mathrm{O}_{2}$ oxidation may occur in the epidermis (25). The presence of albumin and keratin 1,10,11 in vernix is consistent with a proteomics identification of innate immune components (14).

Fibronectin was lowest in vernix and premature skin. It is normally found in the dermoepidermal layer and upper dermis but may also be synthesized by keratinocytes and distributed within the extracellular matrix (26). Fibronectin inhibited terminal differentiation and reduced involucrin in keratinocyte cell cultures (27). It is perhaps not surprising that fibronectin was low in vernix, which lacks corneodesmosomes and a tightly cross-linked SC structure.

All infants transition rapidly from high to low humidity at birth. Transfer from high $(>80 \%)$ to low $(<10 \%)$ humidity leads to decreased skin hydration in the hairless mouse (28). Low humidity $(<10 \%)$ increases epidermal DNA synthesis, suggesting that reduced hydration triggers cell proliferation (29). The mRNA levels for epidermal $\operatorname{IL} 1 \alpha$ are reportedly higher in neonatal animals at low humidity and IL $1 \alpha$ is higher in the upper SC (30). IL $1 \alpha$ and IL $1 \beta$ increased markedly on $\mathrm{d}$ 19 and 20 (d 17, undetectable), decreased significantly at d 21, and increased on $\mathrm{d} 22$ (birth) in the rat (31). TNF $\alpha$ is evident at d 17, decreases on d 21, and increases at birth. The barrier maturation rate is increased in cytokine treated explants, indicating their role in SC development. The higher levels of IL $1 \alpha$ and IL1 $\beta$ in infants versus adults in this study may indicate a similar function for IL1 cytokines, especially when coupled with the increase in IL1 $\alpha$ at low humidity. In contrast to the reports cited above in fetal animals, we observed lower levels of TNF $\alpha$ in preterm and full term infants versus adults. Associated cytokines such as IL6 are increased after SC barrier damage and application of IL6 significantly increases barrier repair in animals (32). IL1RA is increased in inflammatory skin diseases and irritant exposure (33). IL1RA was present in $21 \%$ of our premature infant samples, which may indicate a limited ability to respond to environmental insults.

No attempt was made in this study to investigate the etiology of preterm birth and, in general, the factors causing premature labor are poorly understood. However, preterm labor is strongly associated with chorioamnionitis (34). Although infection may be present at birth, the resulting inflammation may be subclinical (35), prompting the identification of other indicators and precursors. The proinflammatory cytokine $\mathrm{TNF} \alpha$ is higher in decidual tissue in the face of premature labor and intra-amniotic infection (36). Increases in IL1 $\beta$, IL-8, IL-6, and TNF $\alpha$ are associated with term labor
(37). In this study, the higher levels of IL6 and IL8 in premature versus full-term infants are consistent with similar results in dried infant blood samples (38). Higher IL6, IL8, and MCP1 were found in cord blood from lower GA infants compared with full-term neonates (39). IL6 and IL8 were higher in the amniotic fluid of patients with premature labor or preterm premature rupture of membrane (40). The significance of the increased cytokine levels in this study and previous reports is unknown, although specific cytokine profiles may indicate genetic influences (41) and/or clinical conditions associated with a systemic inflammatory response, e.g. bronchopulmonary dysplasia (42). Our results indicate significant correlations among cytokines and important clinical variables which may be important in guiding future research.

The presence of skin surface cortisol in all samples is intriguing. Cortisol exerts various systemic effects, i.e. it stimulates protein metabolism, increases water retention, regulates blood pressure, reduces inflammation, resists stress from infectious agents, physical trauma, and temperature, and reduces the immune response. Cortisol increases are considered to be adaptive, whereas no change indicates a maladaptive reaction (43). The skin contains components of the HPA system and functions as a "decentralized" stress-response system to various environmental stimuli, referred to as the skin stress response system (SSRS) $(6,44)$. The stressors cause release of interleukins, $\mathrm{TNF} \alpha$, etc., which trigger $\mathrm{CRH}$ and POMC. Cortisol is released by hair follicles $(6,44)$. Hair cortisol levels were significantly associated with the number of days on a ventilator in a group of neonatal intensive care patients (45). The identification of surface cortisol in this study is significant and may be a result of the response to various environmental stressors, particularly in the premature infant. Stress due to environmental overcrowding was associated with a delay in skin barrier recovery in mice, an effect attributed to increased production of glucocorticoids (46). Psychological stress decreased epidermal cell proliferation, adversely effected differentiation and decreased the size and density of corneodesmosomes, all of which negatively impact barrier function (47). Stress decreased antimicrobial peptides in the epidermis (animal model), an effect which resulted in more severe skin infections (48).

This study is preliminary and limited to providing a snapshot of biomarker levels within the first week of life. Nevertheless, the results provide insight into the ontogeny of the skin neuroimmune system and this is the first study to measure biomarkers of innate immunity from the outermost epidermal surface (SC) of premature and full-term neonates.

The collective findings suggest that SC barrier maturation is incomplete in both infant groups but to a greater extent in premature infants. The presence of albumin, the inverse correlation of albumin with GA, and the known poorer SC barrier integrity of preterm infants suggest that the elevated levels of albumin and/or cytokines may be due to increased permeability, greater response to environmental, and physiologic stress and/or ease of access to the collection tape. Alternatively, the observed associations of the cytokines and cortisol may be a reflection of a localized stress response; e.g. chorioamnionitis, with local synthesis in the epidermis and/or hair follicles. The 
inflammatory cascade is a dynamic process and temporal profiles in neonates categorized by specific disease processes (42) need to be evaluated. The noninvasive sampling method reported here, coupled with the capability for simultaneous detection and analysis of biomarkers at physiologic levels, offers a promising approach for investigating clinical responses to environmental stressors.

\section{REFERENCES}

1. Agren J, Sjors G, Sedin G 1998 Transepidermal water loss in infants born at 24 and 25 weeks of gestation. Acta Paediatr 87:1185-1190

2. Nonato LB, Lund CH, Kalia YN, Guy RH 2000 Transepidermal water loss in 24 and 25 weeks gestational age infants. Acta Paediatr 89:747-748

3. Slominski A, Wortsman J, Pisarchik A, Zbytek B, Linton EA, Mazurkiewicz JE, Wei ET 2001 Cutaneous expression of corticotropin-releasing hormone (CRH), urocortin, and CRH receptors. FASEB J 15:1678-1693

4. Slominski A, Wortsman J, Tuckey RC, Paus R 2007 Differential expression of HPA axis homolog in the skin. Mol Cell Endocrinol 265-266:143-149

5. Slominski A, Zbytek B, Szczesniewski A, Semak I, Kaminski J, Sweatman T, Wortsman J 2005 Corticotropin-releasing hormone stimulation of corticosteroids production in melanocytes is mediated by ACTH. Am J Physiol Endocrinol Metab 288:E701-E706

6. Ito N, Ito T, Kromminga A, Bettermann A, Takigawa M, Kees F, Straub RH, Paus R 2005 Human hair follicles display a functional equivalent of the hypothalamicpituitary-adrenal axis and synthesize cortisol. FASEB J 19:1332-1334

7. Zouboulis CC, Seltmann H, Hiroi N, Chen W, Young M, Oeff M, Scherbaum WA, Orfanos CE, McCann SM, Bornstein SR 2002 Corticotropin-releasing hormone: an autocrine hormone that promotes lipogenesis in human sebocytes. Proc Natl Acad Sci USA 99:7148-7153

8. Slominski A, Zjawiony J, Wortsman J, Semak I, Stewart J, Pisarchik A, Sweatman T, Marcos J, Dunbar C, Tuckey R 2004 A novel pathway for sequential transformation of 7-dehydrocholesterol and expression of the P450scc system in mammalian skin. Eur J Biochem 271:4178-4188

9. Slominski A 2009 On the role of the corticotropin-releasing hormone signalling system in the aetiology of inflammatory skin disorders. Br J Dermatol 160:229-232

10. Slominski A, Zbytek B, Zmijewski M, Slominski RM, Kauser S, Wortsman J, Tobin DJ 2006 Corticotropin releasing hormone and the skin. Front Biosci 11:2230-2248

11. Walker VP, Akinbi HT, Meinzen-Derr J, Narendran V, Visscher M, Hoath SB 2008 Host defense proteins on the surface of neonatal skin: implications for innate immunity. J Pediatr 152:777-781

12. Nickoloff BJ, Naidu Y 1994 Perturbation of epidermal barrier function correlates with initiation of cytokine cascade in human skin. J Am Acad Dermatol 30:535-546

13. Hendrix SW, Miller KH, Youket TE, Adam R, O'Connor RJ, Morel JG, Tepper BE 2007 Optimization of the skin multiple analyte profile bioanalytical method for determination of skin biomarkers from D-Squame tape samples. Skin Res Technol $13: 330-342$

14. Tollin M, Bergsson G, Kai-Larsen Y, Lengqvist J, Sjovall J, Griffiths W, Skuladottir GV, Haraldsson A, Jornvall H, Gudmundsson GH, Agerberth B 2005 Vernix caseosa as a multi-component defence system based on polypeptides, lipids and their interactions. Cell Mol Life Sci 62:2390-2399

15. Engelke M, Jensen JM, Ekanayake-Mudiyanselage S, Proksch E 1997 Effects of xerosis and ageing on epidermal proliferation and differentiation. Br J Dermatol 137:219-225

16. HogenEsch H, Boggess D, Sundberg JP 1999 Changes in keratin and filaggrin expression in the skin of chronic proliferative dermatitis (cpdm) mutant mice. Pathobiology 67:45-50

17. Visscher MO, Chatterjee R, Munson KA, Pickens WL, Hoath SB 2000 Changes in diapered and nondiapered infant skin over the first month of life. Pediatr Dermatol $17: 45-51$

18. Okah FA, Wickett RR, Pickens WL, Hoath SB 1995 Surface electrical capacitance as a noninvasive bedside measure of epidermal barrier maturation in the newborn infant. Pediatrics 96:688-692

19. Murphy GF, Flynn TC, Rice RH, Pinkus GS 1984 Involucrin expression in normal and neoplastic human skin: a marker for keratinocyte differentiation. J Invest Dermatol 82:453-457

20. Hirao T, Terui T, Takeuchi I, Kobayashi H, Okada M, Takahashi M, Tagami H 2003 Ratio of immature cornified envelopes does not correlate with parakeratosis in inflammatory skin disorders. Exp Dermatol 12:591-601

21. Ekanayake-Mudiyanselage S, Aschauer H, Schmook FP, Jensen JM, Meingassner JG, Proksch E 1998 Expression of epidermal keratins and the cornified envelope protein involucrin is influenced by permeability barrier disruption. J Invest Dermatol 111:517-523
22. Rabilloud T, Asselineau D, Darmon M 1988-1989 Presence of serum albumin in normal human epidermis: Possible implications for the nutrition and physiology of stratified epithelia. Mol Biol Rep 13:213-219

23. Hasse S, Kothari S, Rokos H, Kauser S, Schurer NY, Schallreuter KU 2005 In vivo and in vitro evidence for autocrine $\mathrm{DCoH} / \mathrm{HNF}-1$ alpha transcription of albumin in the human epidermis. Exp Dermatol 14:182-187

24. Yamane Y, Moriyama K, Yasuda C, Miyata S, Aihara M, Ikezawa Z, Miyazaki K 2009 New horny layer marker proteins for evaluating skin condition in atopic dermatitis. Int Arch Allergy Immunol 150:89-101

25. Rokos H, Moore J, Hasse S, Gillbro JM, Wood JM, Schallreuter KU 2004 In vivo fluorescence excitation spectroscopy and in vivo Fourier-transform Raman spectroscopy in human skin: evidence of $\mathrm{H}_{2} \mathrm{O}_{2}$ oxidation of experimental albumin in patients with vitiligo. J Raman Spectroscopy 35:125-130

26. Bernard BA, Robinson SM, Vandaele S, Mansbridge JN, Darmon M 1985 Abnormal maturation pathway of keratinocytes in psoriatic skin. Br J Dermatol 112:647-653

27. Adams JC, Watt FM 1989 Fibronectin inhibits the terminal differentiation of human keratinocytes. Nature 340:307-309

28. Sato J, Denda M, Ashida Y, Koyama J 1998 Loss of water from the stratum corneum induces epidermal DNA synthesis in hairless mice. Arch Dermatol Res 290:634637

29. Denda M, Sato J, Tsuchiya T, Elias PM, Feingold KR 1998 Low humidity stimulates epidermal DNA synthesis and amplifies the hyperproliferative response to barrier disruption: implication for seasonal exacerbations of inflammatory dermatoses. J Invest Dermatol 111:873-878

30. Ashida Y, Ogo M, Denda M 2001 Epidermal interleukin-1 alpha generation is amplified at low humidity: implications for the pathogenesis of inflammatory dermatoses. Br J Dermatol 144:238-243

31. Jiang YJ, Lu B, Crumrine D, Man MQ, Elias PM, Feingold KR 2009 IL-1alpha accelerates stratum corneum formation and improves permeability barrier homeostasis during murine fetal development. J Dermatol Sci 54:88-98

32. Wang XP, Schunck M, Kallen KJ, Neumann C, Trautwein C, Rose-John S, Proksch E 2004 The interleukin-6 cytokine system regulates epidermal permeability barrier homeostasis. J Invest Dermatol 123:124-131

33. Terui T, Hirao T, Sato Y, Uesugi T, Honda M, Iguchi M, Matsumura N, Kudoh K, Aiba S, Tagami H 1998 An increased ratio of interleukin-1 receptor antagonist to interleukin-1alpha in inflammatory skin diseases. Exp Dermatol 7:327-334

34. Goldenberg RL, Culhane JF, Iams JD, Romero R 2008 Epidemiology and causes of preterm birth. Lancet 371:75-84

35. Romero R, Espinoza J, Goncalves LF, Kusanovic JP, Friel LA, Nien JK 2006 Inflammation in preterm and term labour and delivery. Semin Fetal Neonatal Med 11:317-326

36. Casey ML, Cox SM, Beutler B, Milewich L, MacDonald PC 1989 Cachectin/tumor necrosis factor-alpha formation in human decidua. Potential role of cytokines in infection-induced preterm labor. J Clin Invest 83:430-436

37. Keelan JA, Blumenstein M, Helliwell RJ, Sato TA, Marvin KW, Mitchell MD 2003 Cytokines, prostaglandins and parturition-a review. Placenta 24:S33-S46

38. Skogstrand K, Hougaard DM, Schendel DE, Bent NP, Svaerke C, Thorsen P 2008 Association of preterm birth with sustained postnatal inflammatory response. Obstet Gynecol 111:1118-1128

39. Matoba N, Yu Y, Mestan K, Pearson C, Ortiz K, Porta N, Thorsen P, Skogstrand K, Hougaard DM, Zuckerman B, Wang X 2009 Differential patterns of 27 cord blood immune biomarkers across gestational age. Pediatrics 123:1320-1328

40. Hillier SL, Witkin SS, Krohn MA, Watts DH, Kiviat NB, Eschenbach DA 1993 The relationship of amniotic fluid cytokines and preterm delivery, amniotic fluid infection, histologic chorioamnionitis, and chorioamnion infection. Obstet Gynecol 81:941-948

41. Moonen RM, Paulussen AD, Souren NY, Kessels AG, Rubio-Gozalbo ME, Villamor E 2007 Carbamoyl phosphate synthetase polymorphisms as a risk factor for necrotizing enterocolitis. Pediatr Res 62:188-190

42. Ambalavanan N, Carlo WA, D'Angio CT, McDonald SA, Das A, Schendel D, Thorsen P, Higgins RD 2009 Cytokines associated with bronchopulmonary dysplasia or death in extremely low birth weight infants. Pediatrics 123:1132-1141

43. Keenan K, Grace D, Gunthorpe D 2003 Examining stress reactivity in neonates: relations between cortisol and behavior. Child Dev 74:1930-1942

44. Slominski A, Wortsman J, Luger T, Paus R, Solomon S 2000 Corticotropin releasing hormone and proopiomelanocortin involvement in the cutaneous response to stress. Physiol Rev 80:979-1020

45. Yamada J, Stevens B, de Silva N, Gibbins S, Beyene J, Taddio A, Newman C, Koren G 2007 Hair cortisol as a potential biologic marker of chronic stress in hospitalized neonates. Neonatology 92:42-49

46. Denda M, Tsuchiya T 2000 Barrier recovery rate varies time-dependently in human skin. Br J Dermatol 142:881-884

47. Choi EH, Brown BE, Crumrine D, Chang S, Man MQ, Elias PM, Feingold KR 2005 Mechanisms by which psychologic stress alters cutaneous permeability barrier homeostasis and stratum corneum integrity. J Invest Dermatol 124:587-595

48. Aberg KM, Radek KA, Choi EH, Kim DK, Demerjian M, Hupe M, Kerbleski J, Gallo RL, Ganz T, Mauro T, Feingold KR, Elias PM 2007 Psychological stress downregulates epidermal antimicrobial peptide expression and increases severity of cutaneous infections in mice. J Clin Invest 117:3339-3349 